\title{
COMMENTARY \\ Viral bronchiolitis and the detrimental effects of sleep
}

\author{
Daniel Hughes, MD, FRCPC \\ Division of Respirology, Department of Pediatrics, IWK, Halifax, Nova Scotia, Canada
}

\begin{abstract}
Infants recovering from bronchiolitis and no longer requiring supplemental oxygen will have brief episodes of oxygen desaturation during sleep when monitored with pulse oximetry. These episodes can be explained by the inhibition of the tonic activity of the inspiratory muscles during rapid eye movement sleep. Pulse oximetry monitoring should be discontinued, and these infants considered for discharge.
\end{abstract}

lthough there can be no doubt that sleep is beneficial - for adults and children, sleep for some individuals may be detrimental or even fatal, such as falling asleep while driving. The ill effects of sleep may also be apparent in those with sleep disordered breathing, such as obstructive sleep apnea. Many physicians may not be aware of the negative influence of sleep on infants recovering from acute viral bronchiolitis. This is best illustrated by a brief case history.

Baby Jane, a previously healthy six month old infant, had the misfortune to contract a respiratory syncytial virus (RSV) infection and was admitted to hospital. In addition to her respiratory distress and poor feeding, it was her need for supplemental oxygen by nasal prongs that triggered her admission. Her oxygen saturation by pulse oximetry ( $\mathrm{SpO} 2$ ) was $85 \%$ in room air, below the cut-off of $90 \%$ used by the emergency department as one of the criteria for admission. In spite of her cough, tachypnea, subcostal retractions, mild wheeze, and inspiratory crepitations, her course in hospital was uneventful. Supportive care and oxygen were provided, and she was fed orally. On Day 3 of admission, she had minimal respiratory distress and was feeding well. Because she was receiving supplemental oxygen, she remained on continuous $\mathrm{SpO} 2$ monitoring. Anticipating discharge, she had her SpO2 measured while breathing room air, awake, and in her mother's arms. SpO2 was 93\% and oxygen supplementation ceased. She remained connected to the pulse oximeter and the staff noted her SpO2's remained 92-93\% awake but fell to $88-89 \%$ when asleep. She was restarted on supplemental oxygen when sleeping but taken off nasal prongs when awake. Baby Jane remained in hospital two more days until it was determined that her $\mathrm{SpO} 2$ would not fall below $90 \%$ during sleep in room air.

This scenario is repeated frequently during the annual bronchiolitis season and leads some physicians to believe that monitoring by pulse oximetry has led to an increase in length of stay (LOS) for infants with bronchiolitis ${ }^{1,2,3}$.

Pulse oximetry is a simple non-invasive monitoring device, used initially in acute care settings - emergency departments, intensive care units, and operating rooms since the early 1980's. Known as the "fifth vital sign" pulse oximetry is now widely used on pediatric wards in patients at risk for hypoxemia ${ }^{4}$.

Acute viral bronchiolitis is the leading cause of hospital admission in infants less than 12 months of age. Most admitted patients are hypoxemic and monitored with pulse oximetry. Admission rates for bronchiolitis have increased in recent years and some physicians believe this is associated with the overdiagnosis of borderline hypoxemia by pulse oximetry ${ }^{5,6}$.

Guidelines for pulse oximetry, spot checks versus continuous monitoring, and acceptable $\mathrm{SpO} 2$ levels have varied. SpO2 minimums have fallen from $94 \%^{7}$ to the generally accepted levels of $90 \%$. The American Academy of Pediatrics (AAP) ${ }^{8}$, Royal Children's Hospital (Melbourne) ${ }^{9}$, and Canadian Pediatric Society $(\mathrm{CPS})^{10}$ recommend $90 \%$ while the UK National Institute for Health and Care Excellence (NICE) recommends $92 \%{ }^{11,12}$.

Focusing on the circumstances around the time of discharge and considering that as many as $25 \%$ of infants with bronchiolitis will have an increased LOS as a result of a perceived need for oxygen $^{2}$, what do the guidelines recommend? The AAP does not specify discharge criteria, simply stating, "clinicians may choose not to administer supplemental oxygen if the oxyhemoglobin saturation exceeds $90 \%$. . " ${ }^{8}$. The guideline does recognize that transient desaturation is a normal phenomenon in healthy infants where as many as $60 \%$ will exhibit a transient oxygen desaturation below $90 \%$ to values as low as $83 \%{ }^{13}$. The Royal Children's Hospital guideline recommends discontinuing oxygen when $\mathrm{SpO} 2$ is persistently $\geq 90 \%$ but also state stopping SpO2 monitoring if not requiring oxygen for 2 hours 9 . They note that "infants with bronchiolitis will have brief episodes of mild/moderate desaturations to levels less than 90\%. These brief desaturations are not a reason to commence oxygen therapy." The CPS, while not specifically focusing on discharge, does recognize that transient oxygen desaturations can occur and that continuous monitoring may lead staff to react to normal transient dips in SpO2 and restart oxygen therapy ${ }^{10}$.

The only guideline to mention sleep is $\mathrm{NICE}^{11}$. For discharge, NICE recommends that infants ". . . . have maintained oxygen saturation over $92 \%$ in air for four hours, including a period of sleep." This recommendation, unfortunately, will lead to 
the re-institution of oxygen therapy when brief desaturations less than 92\% occur. In August 2019 NICE proposed that they needed to update their guideline stating that "the update will focus on safe and effective levels of oxygen saturation when discharging patients"11.

Recognizing that healthy infants and infants with bronchiolitis can have brief oxygen desaturations, how might this be related to sleep?

Tonic activity of the intercostal muscles and diaphragm are important for the maintenance of functional residual capacity (FRC) in infants. During rapid eye movement (REM) sleep, the tonic activity of inspiratory muscles is inhibited and in healthy newborns the thoracic gas volume is reduced $31 \%{ }^{14}$. The lack of tone in the intercostal muscles may cause the chest distortion to be readily apparent in the infant with a compliant chest wall. The fall in FRC leads to a subsequent drop in $\mathrm{SpO} 2$ detected by the pulse oximeter.

Seeking to determine whether episodes of desaturation prior to discharge in patients with bronchiolitis were associated with sleep, Kaditis et al performed continuous SpO2 measurements from 10 $\mathrm{pm}$ to $8 \mathrm{am}^{15}$. They compared patients recovering from bronchiolitis with patients without lung disease and those with chronic partial upper airway obstruction without lung disease. Prior to discharge, infants with bronchiolitis exhibited low basal SpO2's and multiple abrupt SpO2 drops during the night. The authors speculated that the low SpO2's reflected the sleep associated reduction in FRC and deterioration in ventilation-perfusion (V/Q) mismatch. They also commented on the frequent clinical scenario of re-instituting oxygen administration during sleep in infants with bronchiolitis who have been weaned off oxygen while awake, the point of this article. We have physiological evidence for drops in SpO2's during sleep in infants with bronchiolitis, a phenomenon that also occurs in adolescents with asthma ${ }^{16,17}$.

What might be possible solutions to address this issue? The Choosing Wisely campaign recommends avoiding continuous pulse oximetry in children admitted for respiratory illnesses who are not using supplemental oxygen ${ }^{18}$. The AAP clinical practice guideline on bronchiolitis suggests similar advice ${ }^{8}$. The message is that continuous pulse oximetry is more likely to detect transient oxygen desaturations of uncertain significance. The CPS guidelines also recommend intermittent monitoring except for high risk patients ${ }^{10}$.

This author suggests one step further. When an otherwise healthy infant is recovering from bronchiolitis and (1) has minimal respiratory symptoms, (2) is feeding satisfactorily (75\% of normal intake), (3) is maintaining SpO2's $\geq 90 \%$ when awake in room air for more than 2 hours and not requiring supplemental oxygen, and (4) there are no social/family issues to prevent discharge, discontinue pulse oximetry monitoring and send the patient home.

\section{References}

1. Quinonez R, Coon E, Schroeder A, Moyer V. When technology creates uncertainty: pulse oximetry and overdiagnosis of hypoxaemia in bronchiolitis. BMJ. 2017;358:j3850

2. Schroeder A, Marmor A, Pantell R, Newman T. Impact of pulse oximetry and oxygen therapy on length of stay in bronchiolitis hospitalizations. Arch Pediatr Adolesc Med. 2004;158:527-530

3. Unger S, Cunningham S. Effect of oxygen supplementation on length of stay for infants hospitalized with acute viral bronchiolitis. Pediatrics. 2008;121:470-475

4. Sinha I, Mayell S, Halfhide C. Pulse oximetry in children. Arch Dis Child Educ Pract Ed. 2014;99:117-118

5. Meissner HC. Viral bronchiolitis in children. N Engl J Med. 2016;374:62-72

6. Wainwright C. Acute viral bronchiolitis in children - a very common condition with few therapeutic options. Paediatr Resp Reviews. 2010;11:39-45

7. Adcock P, Sanders C, Marshall G. Standardizing the care of bronchiolitis. Arch Pediatr Adolesc Med. 1998;152:739-744

8. Clinical Practice Guideline: The diagnosis, management, and prevention of bronchiolitis. Pediatrics. 2014;134:e1474-502

9. Bronchiolitis. [www.rch.org.au>clinicalguide>guideline_index>bronchiolitis] (Accessed on Feb. 23, 2020).

10. Bronchiolitis: recommendations for diagnosis, monitoring and management of children 1-24 months of age. [www.cps.ca/en/ documents/position/bronchiolitis] (Accessed on Feb. 23, 2020).

11. Bronchiolitis in children: diagnosis and management. NICE guideline (N69) June 2015 [www.nice.org.uk/guidance/ng9] (Accessed on Feb. 23, 2020).

12. Ricci V, Delgado Nunes V, Murphy MS, Cunningham S. Guideline Development Group and Technical Team. Bronchiolitis in children: summary of NICE guideline. BMJ. 2015;350:h2305

13. Hunt C, et al. Longitudinal assessment of hemoglobin oxygen saturation in healthy infants during the first 6 months of age. J Pediatr. 1999;134:580-6

14. Henderson-Smart D, Read D. Reduced lung volume during behavioral active sleep in the newborn. J Appl Physiol:Respirat Environ Exercise Physiol. 1979;46(6):1081-85

15. Kaditis A, et al. Infants with viral bronchiolitis demonstrate two distinct patterns of nocturnal oxyhaemoglobin desaturation. Acta Paediatrica. 2015;104:e106-e111

16. Tabachnik E, Muller N, Levison H, Bryan A. Chest wall mechanics and pattern of breathing during sleep in asthmatic adolescents. Am Rev Respir Dis. 1981;124:269-273

17. Chipps B, et al. Nocturnal oxygen saturation in normal and asthmatic children. Pediatrics. 1980;65:1157-1160

18. Quinonez R, et al. Choosing wisely in pediatric hospital medicine: five opportunities for improved healthcare value. Journal of Hospital Medicine. 2013;8:479-485 